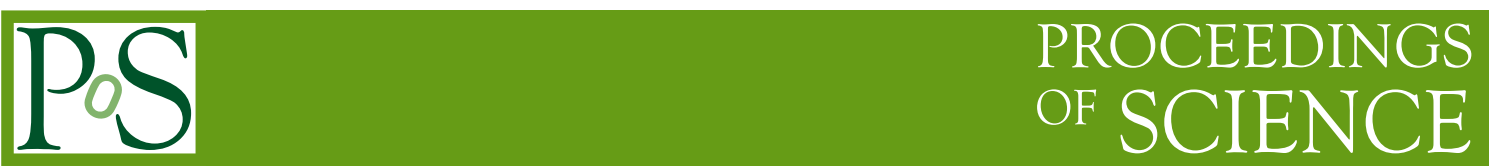

\title{
Measurements of Cosmic Ray Electrons and Positrons: Current Status and Perspectives
}

\author{
Alexander Moiseev ${ }^{1}$ \\ CRESST/NASA/GSFC and University of Maryland, College Park \\ NASA/GSFC, code 661, Greenbelt, MD 20771 \\ E-mail: alexander.a.moiseev@nasa.gov \\ This paper reviews the existing results on high-energy cosmic ray electron (CRE) measurements \\ and their interpretations. It includes results on inclusive electron spectrum, positron fraction in \\ CRE, and CRE anisotropy, with the focus on recent Fermi LAT and PAMELA measurements. \\ Future perspectives in CRE measurements are presented too.
}

Science with the New Generation of High Energy Gamma-ray experiments, 10th Workshop (Scineghe2014)

04-06 June 2014

Lisbon - Portugal

1

Speaker 


\section{Introduction: Why electrons?}

The problem of cosmic ray (CR) origin has been puzzling scientists for decades. Galactic magnetic field diverts charged cosmic rays and therefore does not allow to trace their origination point by measuring arrival direction (the Larmor radius for $1 \mathrm{TeV}$ particle is $\sim 2 \mathrm{x}$ $10^{-4} \mathrm{pc}$ assuming galactic magnetic field strength $\left.\mathrm{B}=4 \mu \mathrm{G}\right)$. It is believed that the majority of $\mathrm{CR}$ are produced in pulsar systems such as SNR and PWN, but this hypothesis has never been directly confirmed. Due to their low mass, high energy cosmic ray electrons (CRE) ${ }^{2}$ rapidly lose their energy during propagation through local magnetic fields via inverse Compton and synchrotron radiation with strong energy dependence $\mathrm{dE} / \mathrm{dt} \sim \mathrm{E}^{2}$ and thus cannot travel far from their sources. The characteristic energy loss time for a few $\mathrm{TeV}$ electrons is $\sim 10^{5}$ years, and corresponding travel distance is $\sim 1 \mathrm{kpc}$ (for the diffusion coefficient $\mathrm{D}=10^{28} \mathrm{~cm}^{2} / \mathrm{s}$ ), so they can serve as a probe of nearby Galactic sources and propagation. From the other point, measured spectrum of CRE and their charge composition can contribute to the modeling of their possible sources (such as SNR), especially if the source can be identified by its estimated age and distance. All this makes CRE a unique tool to probe nearby Galactic space (Fig.1).

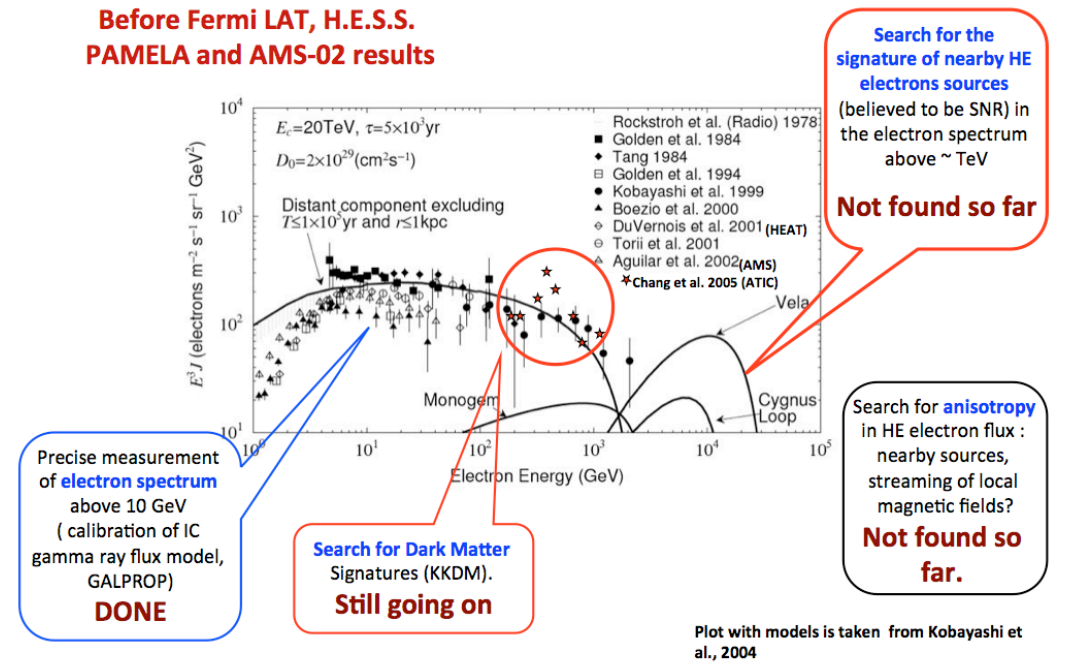

Figure 1. What can be learned from high-energy cosmic-ray electrons?
CRE have been studied for several decades, starting from high-altitude balloon flights, and currently in space missions. Recent results on CRE from the ATIC [1], PAMELA [2], PPBBETS [3], and Fermi LAT dramatically increased an interest to this topic. All the measurements can be divided in three

major groups: inclusive CRE spectrum, separate electron and positron spectra and their ratio, and anisotropy of their arrival. Negative electrons are believed to be produced in sources, but positrons are believed to be mainly secondary produced in CR-interstellar media interactions. Currently measured electron-to-positron ratio clearly departs from that expected, raising intriguing interpretations.

\footnotetext{
2 "electrons" means both, negatrons and positrons, unless specified as electrons and positrons separately
} 


\section{Current results on inclusive electron spectrum}

Before the results from AMS-01 (flown on Space Shuttle Discovery in 1998), published in 2002, all measurements of CRE were made in high-altitude balloon flights. The spectra were consistent with the single-index power law spectrum with an index of approximately -3.3 [6], and was interpreted as a background from a conventional homogeneous distribution of cosmic ray sources. AMS-01 spectrum was also consistent with previous measurements [7] (Fig.2, upper left panel). However, the results from PPB-BETS [3] and ATIC [1] reported in 2008 an intriguing excess of electrons in the range $300-700 \mathrm{GeV}$ compared to that "conventional" single-index power law spectrum (Fig.2, upper right panel). That evident spectral feature has been discussed in numerous papers, suggesting the presence of additional component with either astrophysical (pulsar or super-novae remnant) or exotic, such as dark matter, origin ( see e.g. [8] and references therein). The H.E.S.S. team reported in 2009 a spectrum that sharply steepens above $\sim 900 \mathrm{GeV}$ [9], a very important result which possibly indicates an absence of sources of electrons above 1-2 TeV within the nearby Galaxy of 300-400 pc.
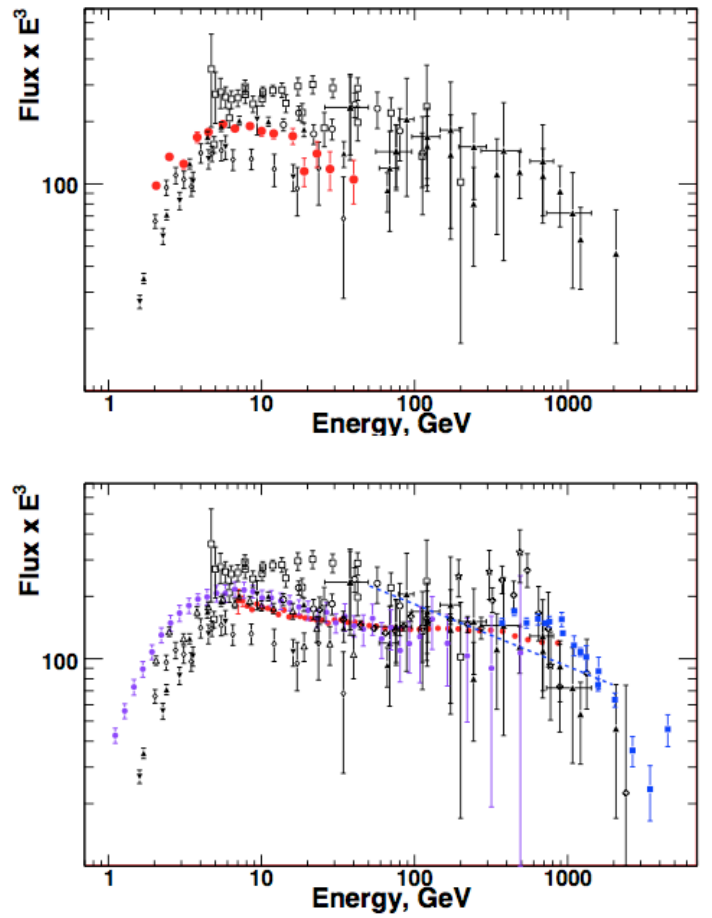

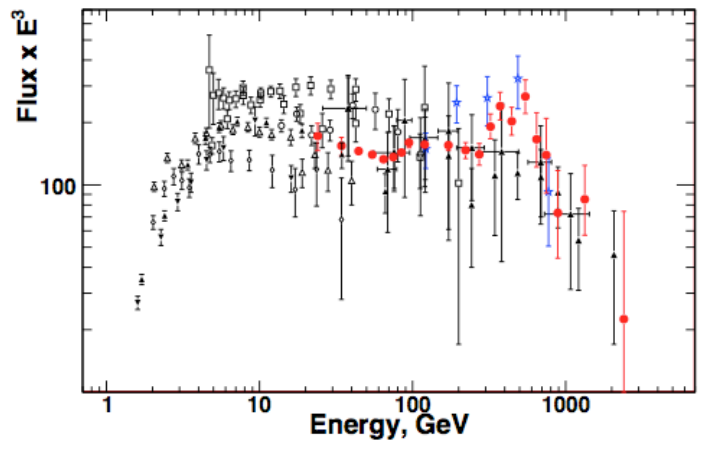

Fig.2. Inclusive electron spectra from different experiments:

Upper left panel: early balloon experiments ( $\mathrm{J}$. Nishimura 80, T. Kobayashi 99, K.-K. Tang 84, R. Golden 84, 94, M. Boezio 00, S. Torii 02, M. deVernois 01). Red circles: first space measurement by AMS-01 02)

Upper right panel: added PPB-BETS (blue stars) and ATIC (red circles)

Bottom panel: Current results. Red circles: Fermi LAT, blue squares: H.E.S.S., both papers, violet circles: PAMELA. Dashed line: power law with earlier measured index $-3-3$

The most accurate and almost statistically error-free measurement of the CRE spectrum was provided by the Fermi Large Area Telescope in 2009 (Fermi LAT) [4,5]. The Fermi Gammaray Space Telescope was launched on June 11, 2008 on $565 \mathrm{~km}$ Earth circular orbit. The LAT is a pair-conversion gamma-ray telescope with field-of-view $2.4 \mathrm{sr}$, effective area (for photons) of $\sim 8,000 \mathrm{~cm}^{2}$ at $1 \mathrm{GeV}$, and energy range from $20 \mathrm{MeV}$ to more than $300 \mathrm{GeV}$ [10]. It consists of 16 identical towers that provide conversion of a gamma-ray into $\mathrm{e}^{+} \mathrm{e}^{-}$pair and determine its arrival direction (18 double-plane Si-strip Tracker) and energy (segmented hodoscopic CsI Calorimeter). The instrument is covered by a segmented AntiCoincidence Detector, which 
rejects the charged particle background. The electronic system includes flexible and robust hardware trigger along with software filters.

Although the LAT is a gamma-ray telescope, in early stage of its design it was recognized that intrinsically the LAT is also a capable electron spectrometer [11]. The facts that the most of the time the LAT operates in scanning mode and its wide aperture make it particularly suitable for measurement of the isotropic cosmic radiation (as CRE flux is assumed to be). Large effective geometric factor $\left(2.7 \mathrm{~m}^{2} \mathrm{sr}\right.$ at $\left.50 \mathrm{GeV}\right)$ secures very high statistics. The LAT cannot distinguish electrons from positrons by its detection technique, but in the next section it will be shown that it can be done using the Earth magnetic field.

The resulting spectrum obtained by Fermi LAT during the first year of operation, as well as all the details of the analysis, are given in [5]. It contains $\sim 8$ million electron events in the energy range of $7 \mathrm{GeV}$ to $1 \mathrm{TeV}$. Within the systematic errors, the entire spectrum can be fitted by a power law with spectral index within the interval $3.08 \pm 0.05$. The spectrum is significantly harder than that reported by previous experiments. At the same time, the spectrum suggests some flattening at 70-200 GeV with noticeable excess above $200 \mathrm{GeV}$ as compared to powerlaw spectral fit, and can be fitted by a twice broken power law injection spectrum (Fig.2, bottom panel).

In their second paper on CRE, H.E.S.S. presented the spectrum extended down to $340 \mathrm{GeV}$, which is very challenging for the ground-based Cherenkov telescope technique [12]. The spectrum is consistent with Fermi LAT, and also free of any noticeable spectral feature like PPB-BETS or ATIC.

Precision measurement of the CRE spectrum was provided by the satellite-born experiment PAMELA [13] in 2011. PAMELA is a magnetic spectrometer designed by the collaboration of Italy, Russia, Germany and Sweden, which provided groundbreaking results on the positron fraction in CRE (to be given in next section). The electron spectrum, measured by PAMELA, collected for 3.5 years of operation, covers the energy interval from 1 to $625 \mathrm{GeV}$ and can be described with a single-index power-law spectrum with spectral index -3.180 .05 (above the energy of $\sim 30 \mathrm{GeV}$, where electrons are influenced by the solar wind). No significant spectral features were observed, and spectrum is consistent with that of Fermi LAT (Fig.2, bottom panel).

\section{Measurement of the charge ratio in CRE flux}

Positrons in cosmic rays were believed to be secondary and produced in interactions of primary cosmic rays with interstellar gas [14,15]. This process results in a positron fraction decreasing with energy. Measurements of positron fraction in statistically limited balloon experiments, starting in early-60, and up to 1996, showed fraction decreasing up to $\sim 3-4 \mathrm{GeV}$, but starting increasing at higher energy [14 and references therein] (Fig.3, left panel). It was in strong disagreement with their assumed secondary origin, but in that experiments the excessive positrons could be due to unaccounted protons, potentially contaminating the result. Real breakthrough was made by PAMELA, which measured positron fraction in the energy range from 1.5 to $100 \mathrm{GeV}$ with high confidence [2]. It was found that the positron fraction increases 

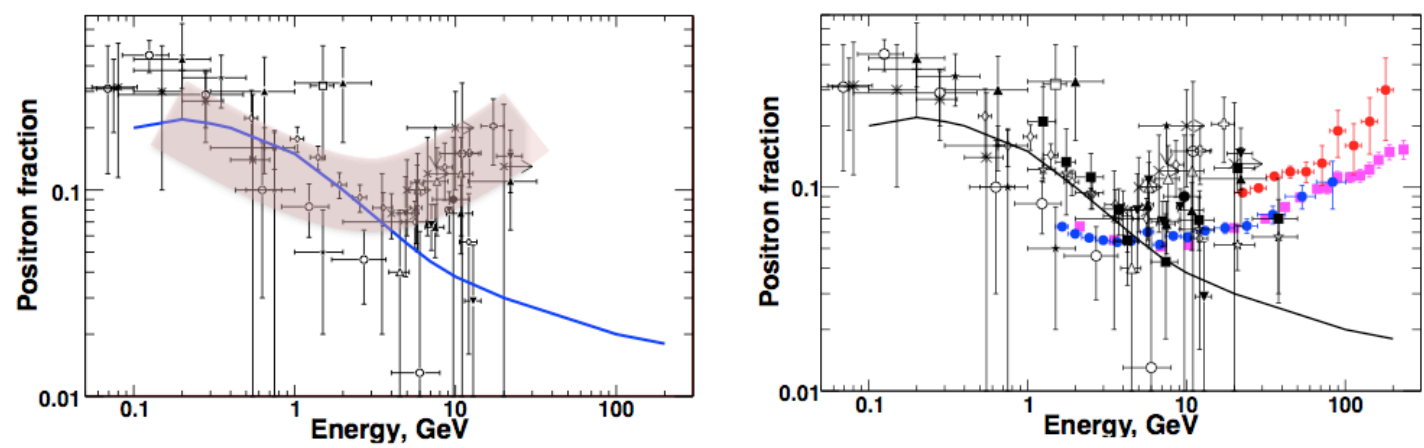

Figure 3. Results on positron fraction. Left panel: early results at the end of 1996 (DeShone 64, Hartman 65, 67, Fanselow 69, Agrinier 69, Dagherty 75, Buffington 75, Muller 87, Golden 87, 94, 96, Clem 96, Barbellini 96, Boezio 00). Shaded area shows the trend. Right panel: current results. Blue circles - PAMELA [2], red circles - Fermi LAT [16], and magenta squares - AMS-02 [17]. Solid lines in both panels show positron fraction if all positrons were secondary [15], calculated with GALPROP

sharply over much of that range, in a way that appears to be completely inconsistent with assumed secondary mechanism of their production (Fig.3, right panel).

Soon after that Fermi LAT team reported their results on positron fraction. Fermi LAT does not have a magnet, and electrons and positrons were separated by using Earth's shadow, which is offset in opposite directions for opposite charges due to Earth's magnetic field. It was a delicate analysis, requiring careful and accurate calculation of every event trajectory in Earth magnetic field, using its high-precision model [16]. As a result, the CR positron and electron spectra separately and their ratio were measured between 20 and $200 \mathrm{GeV}$. It was found that the positron fraction increases with energy, consistent with results reported by PAMELA.

Recently, AMS-02 presented their result on positron fraction with high accuracy, extending energy up to $250 \mathrm{GeV}$ [17]. Fraction increase with energy, reported by PAMELA and Fermi LAT was confirmed, and the results are in agreement (Fig.3, right panel).

\section{Search for CRE anisotropy}

Search for arrival anisotropy of cosmic rays has always been an appealing subject: if found, it could potentially point to the cosmic ray source. Fermi LAT has detected more than 1.6 million cosmic ray electrons with energies above $60 \mathrm{GeV}$ during its first year of operation, so it was natural to make an attempt of searching for their arrival anisotropy (it is necessary to start the analysis from higher energy to exclude the effect of solar modulation). The search for anisotropy for electrons is challenging because the Larmor radius $\left(\sim 3 \times 10^{-5}\right.$ for $100 \mathrm{GeV}$ electron) is considerably smaller than the typical distance to a nearby source. However, if found it can provide unique information on the sources of CRE and propagation environment. Another complicated issue is that the dipole anisotropy, caused by a sole CRE source, would potentially be possible to detect, but in the case of several nearby CRE sources (multipole anisotropy) it would be significantly more difficult, or simply impossible. Anyways, Fermi LAT team performed this search for anisotropies of angular scale extending from $\sim 10^{\circ}$ up to $90^{\circ}$, for electrons with energy from $60 \mathrm{GeV}$ to $480 \mathrm{GeV}$ [18]. Two independent techniques were used, 
and each resulted in a null result. The upper limits for a dipole anisotropy ranged from $\sim 0.5 \%$ to $\sim 10 \%$ depending on the electron energy.

\section{Possible interpretation of the results}

There have been numerous papers published since 2008, suggesting different scenarios to explain the results. PPB-BETS and ATIC ignited appearance of the large amount of papers, many of which discussing possible dark matter origin of reported spectral feature. The new storm of interpretation papers came after the PAMELA's result on positron fraction and H.E.S.S. and Fermi LAT results on inclusive electron spectrum (see [8] and references therein). Published Fermi LAT 1-year spectrum has a noticeable excess above $200 \mathrm{GeV}$ as compared to power-law spectral fit [5]. This spectrum can be explained within a conventional model by adjusting the injection spectra, e.g. assuming it to be twice-broken power-law with spectral index 1.6/2.5 below and above $4 \mathrm{GeV}$, and 5 above $2 \mathrm{TeV}$ (Fig.4, bottom panel). However, PAMELA's positron fraction cannot be explained by only adjusting injection spectra and propagation parameters and so added critical constrains. A viable interpretation [8] can include an additional component of $\mathrm{e}^{+} \mathrm{e}^{-}$pairs with a hard spectrum. Fig 4. illustrates such a scenario
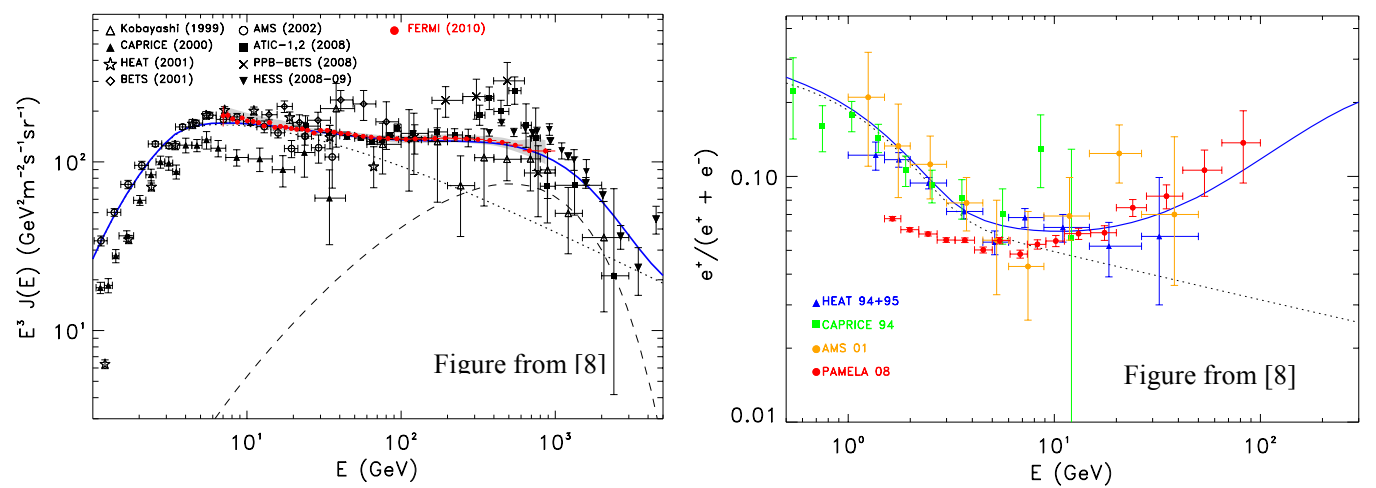

Figure 4. Fermi LAT inclusive spectrum (upper left panel) and Pamela positron fraction (upper right panel) fitted with an additional component (shown by dashed lines). Dotted lines correspond to the spectra without additional component. Bottom panel: Fermi LAT spectrum, fitted with conventional GALPROP model (see parameters in the text).

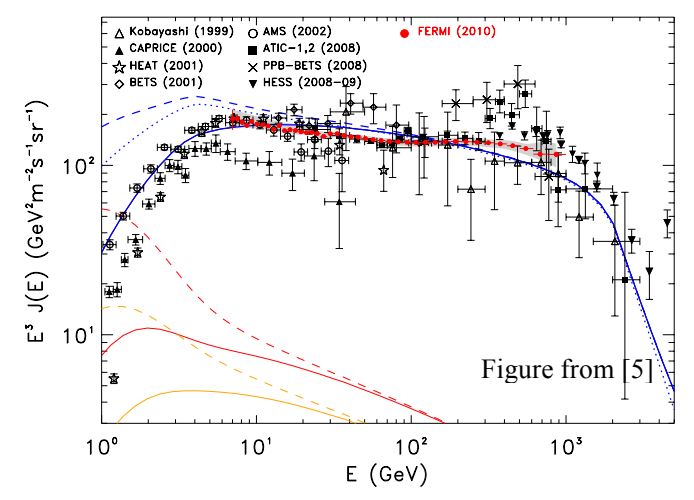

where a good fit to both - the Fermi LAT electron inclusive spectrum (upper left panel) and the PAMELA positron fraction (upper right panel) is achieved by including an additional component of $\mathrm{e}^{+} \mathrm{e}^{-}$with an injection spectral index 1.5 and an exponential cutoff. 
The nature of such an additional component has been discussed in numerous recent papers. Suggested scenarios can be divided into three basic groups: $\mathrm{e}^{+} \mathrm{e}^{-}$component is produced a) in astrophysical objects such as pulsar systems, b) in dark matter, c) by other astrophysical effects. However, all of them assume that the $\mathrm{e}^{+} \mathrm{e}^{-}$component has a harder spectrum than the pure $\mathrm{e}^{-}$ flux. It is the most likely that the CRE only experiments will not be able to prove one or another mechanism, and a multi-messenger approach is necessary.

\section{Future perspectives in CRE measurements}

Fermi LAT. Currently the team is finishing the updated analysis of CRE with improved event reconstruction algorithm (Pass 8), where the systematic errors are better understood and energy reconstruction is improved and extended to above $1 \mathrm{TeV}$. The updated spectrum will include statistics for $6+$ years on the orbit, with expected $\sim 10,000$ electrons only above $1 \mathrm{TeV}$ (depending on the spectrum to be measured). Such a high statistics will make possible to update the anisotropy search, as well as to perform a search for the fine spectral structure.

AMS-02. It is expected that the results on the inclusive electron spectrum from AMS-02 to appear soon. Also, the positron fraction measurement will be extended to higher energy.

PAMELA. This instrument is in successful operation on orbit for more than 8 years, since June 15,2006 . We may expect new and updated results from this beautiful experiment.

CALET (CAlorimetric Electron Telescope) [19] is a Japanese-led international mission (in cooperation with US and Italy) for investigation of high-energy cosmic ray electrons, protons and nuclei. It is scheduled for the launch in the first half of 2015 and will be installed in Japanese Module (KIBO) of International Space Station. CALET has superior energy resolution and excellent separation of hadrons, nuclei and gamma-rays, with its main objective to accurately measure CRE spectrum up to unachievable before $20 \mathrm{TeV}$. It will perform a search for a spectral structure at very high energy, where potential sources of CRE such as Vela, Monogem and Cygnus Loop remnants would leave their signatures [23].

GAMMA-400 [20] is a future Russian-led high-energy gamma-ray mission, currently planned for 2019. Its objectives are to follow and deepen the findings of Fermi LAT with unprecedented for such studies energy and angular resolution at energy above $10 \mathrm{GeV}$, with the main aim of searching for dark matter. However, similarly to Fermi LAT, it will be capable to measure CRE, also with very high energy resolution and acceptance, so we can expect more results on highenergy CRE from GAMMA-400.

PEBS is a dedicated balloon experiment, which could provide a competitive measurement of the cosmic-ray electron and positron flux (separately) from 1 to $500 \mathrm{GeV}$. This spectrometer is based on a scintillating fiber tracker with Silicon Photomultiplier readout and a permanent magnet. It also contains the Time-of-flight, Transition Radiation Detector and a Calorimeter, which all together provide the proton rejection of $10^{6}$ and better. The instrument team completed the design, passed beam tests and prototyping. This is a potential experiment in the nearest future, but currently is on hold.

DAMPE [24] is a Chinese space telescope for high-energy gamma-rays, electrons and cosmic rays, with potential launch in 2015-2016. It contains plastic AntiCoincidence detector, Si-W 
tracker-converter, and $\mathrm{BGO}$ imaging calorimeter, $31 \mathrm{X}_{0}$ thick. It will cover energy range for electrons from $5 \mathrm{GeV}$ to $10 \mathrm{TeV}$, with $\mathrm{dE} / \mathrm{E} \sim 1 \%$ at $800 \mathrm{GeV}$, and geometric factor of $0.3 \mathrm{~m}^{2} \mathrm{sr}$ (see [21] for more details)

CREST [22]: Cosmic Ray Electron Synchrotron Telescope utilizes unique and challenging technique of detecting synchrotron radiation of primary CR electron as it passes through Earth's magnetic field. It is designed for the Antarctic long duration balloon flight, and is able to detect very high-energy CRE above a few TeV with geometric factor approaching $\sim 10-20 \mathrm{~m}^{2} \mathrm{sr}$ at energy above $10 \mathrm{TeV}$. This is probably the only technique that can allow the measuring of such a high energy with reasonably large geometric factor.

\section{Summary}

It has been a real breakthrough during last few years in cosmic-ray electrons: ATIC, H.E.S.S., PAMELA, Fermi LAT, and finally AMS-02. New quality data have made it possible to start quantitative modeling. However, it appears that with the new data we have more puzzles than before, and multi-messenger campaign is needed, involving electrons, positrons, gammarays, X-rays, radio, neutrino. With CRE experiments we may be coming close to the first direct detection and identification of cosmic-ray source(s). Now we can discuss not only the origin of CRE, but also constrains of pulsar models based on these results. It is viable that we are dealing with at least two distinct mechanisms of "primary" electron and positron production. One produces softer spectrum of negative electrons, and the other produces a harder spectrum of both electrons and positrons, or maybe selective production of positrons only? Exotic, e.g. dark matter, origin is not ruled out. Critical results on the positron fraction at higher energy are expected soon from AMS-02.

\section{References}

[1] J. Chang et al., Nature (London) 456, 362 (2008)

[2] O. Adriani et al., Nature (London) 458, 607 (2009)

[3] S. Torii et al., arXiv:0809.0760

[4] A. Abdo et al., Phys. Rev. Lett. 102, 181101 (2009)

[5] M. Ackermann et al., Phys. Rev. D 82, 092004 (2010)

[6] T. Kobayashi et al., in Proceedings of the $26^{\text {th }} I C R C$, Salt Lake City, 1999, http://adsabs.harvard.edu/abs/1999ICRC..3..61K

[7] M. Aguilar et al., Phys. Rep. 366, 331 (2002)

[8] D. Grasso et al., Astroparticle Physics 32, 140 (2009) 
[9] F. Aharonian et al., Phys. Rev. Letters 101, 261104 (2008)

[10] W.B. Atwood et al., Astrophys. J. 697, 1071 (2009)

[11] A. A. Moiseev et al., in Proceedings of the $30^{\text {th }}$ ICRC, Merida, 2, 449 (2007)

[12] F. Aharonian et al., Astronomy and Astrophysics 508, 561 (2009)

[13] O. Adriani et al., Phys. Rev. Lett. 106, 201101 (2011), P. Picozza and L. Marcelli, Astroparticle Physics 53, 160 (2014)

[14] R.J. Protheroe, Astrophys. J. 254, 391 (1982)

[15] I.V. Moskalenko and A. W. Strong, Astrophys. J. 493, 694 (1998)

[16] M. Ackermann et al., Phys. Rev. Lett. 108, 011103 (2012)

[17] M. Aguilar et al., Phys. Rev. Lett. 110, 141102 (2013)

[18] M. Ackermann et al., Phys. Rev. D 82, 092003 (2010)

[19] S. Torii, Nuclear Instruments and Methods A630, 55 (2011)

[20] A.M. Galper et al., http://arxiv.org/abs/1306.6175 (2013), O. Adriani, these Proceedings

[21] G. Ambrosi, these Proceedings

[22] S. Coutu, in Proceedings of the 33th ICRC, Rio de Janeiro, 2013

[23] T. Kobayashi et al., ApJ 601, 340 (2004)

[24] J. Wu and J. Chang, in the Proceedings of 33th ICRC, Rio de Janeiro, 2013 